\title{
Inversion of crater morphometric data to gain insight on the cratering process
}

\author{
ROBERT R. HERRICK ${ }^{1 *}$ AND SUZANNE N. LYONS ${ }^{2 \dagger}$
}

\author{
'Lunar and Planetary Institute, 3600 Bay Area Boulevard, Houston, Texas 77058, USA \\ ${ }^{2}$ Department of Geology and Geophysics, Texas A\&M University, College Station, Texas 77843, USA \\ †Present address: Institute of Geophysics and Planetary Physics, \\ Scripps Institution of Oceanography, La Jolla, California 92093-0220, USA \\ *Correspondence author's e-mail address: herrick@lpi6.jsc.nasa.gov
}

(Received 1997 May 19; accepted in revised form 1997 October 23)

\begin{abstract}
In recent years, morphometric data for Venus and several outer planet satellites have been collected, so we now have observational data of complex craters formed in a large range of target properties. We present general inversion techniques that can utilize the morphometric data to quantitatively test various models of complex crater formation. The morphometric data we use in this paper are depth of a complex crater, the diameter at which the depth-diameter ratio changes, and onset diameters for central peaks, terraces, and peak rings. We tested the roles of impactor velocities and hydrostatic pressure $v s$. crustal strength, and we tested the specific models of acoustic fluidization (Melosh, 1982) and nonproportional growth (Schultz, 1988). Neither the acoustic fluidization model nor the nonproportional growth in their published formulations are able to successfully reproduce the data. No dependence on impactor velocity is evident from our inversions. Most of the morphometric data is consistent with a linear dependence on the ratio of crustal strength to hydrostatic pressure on a planet, or the factor $c / \rho g$.
\end{abstract}

\section{INTRODUCTION}

Crater morphometry, the quantitative description of the shape of impact craters, has always played a key role in understanding the cratering process. One of the key arguments used to support the impact origin of lunar craters was that they were morphometrically similar to terrestrial explosion craters (Baldwin, 1949). Complex impact craters, craters with such features as a flat floor, a central peak, and wall terraces, have never been created in common geologic materials in the lab or with large explosions. At present, only the morphometry of impact craters on the solid bodies of the solar system can provide data on how various target and impactor properties affect complex crater formation. Until recently, morphometric data for fresh complex craters existed only for the Moon, Mars, and Mercury (e.g., Hale and Head, 1979, 1980, 1981; Malin and Dzurisin, 1978; Pike, 1977, 1980a, 1988; Pike and Spudis, 1987; Smith and Hartnell, 1978; Wood, 1980; Wood and Andersson, 1978). Unfortunately, these bodies provide data for a fairly limited range of target properties. As a consequence, past attempts at explaining interplanetary differences in craters have necessarily been limited to a forward-modeling approach, where a model is considered adequate if it fits the data within error tolerances. A well-known example of this approach is Pike's (1980a, 1988) demonstration that a line with slope $1 / g$, where $g$ is surface gravitational acceleration, fits within error bars the simple-to-complex transition diameter for the Moon, Mercury, Mars, and Earth.

The recent addition of crater morphometric data for Venus (Herrick and Phillips, 1994; Sharpton, 1994; Alexopoulus and McKinnon, 1994; Herrick et al., 1996) and the icy satellites (Schenk, 1989, 1991) greatly extends the range of surface gravities and target compositions for which data exist. This additional data makes the inverse approach a feasible method for determining the factors controlling interplanetary differences in crater morphometry and for evaluating various complex-crater formation models. In the inverse approach, a general model is presented and the data are used to invert for any unknown parameters in the model. Standard inversion techniques provide a structured framework for comparing models, incorporating data with errors, and determining ranges of acceptable parameters. Our purpose in this paper is twofold. We will attempt to demonstrate the advantages of the inverse method for making quantitative evaluations of models of the cratering process. In the process, we will test some general concepts regarding the effects of projectile velocity, target surface gravity, and crustal strength on complex-crater formation, and we will test two previously proposed models.

\section{INVERSION TECHNIQUES}

To illustrate what types of data are necessary, what kinds of models can be tested, and what can be learned about each model, here we briefly review some of the key aspects of linear and nonlinear inversion techniques (excellent, more detailed reviews are in Menke, 1989 and Lines and Treitel, 1984). The first step in using inversion techniques to evaluate a model for some aspect of the cratering process is to quantify the model so that it predicts a set of measurable quantities, i.e., measurement $d_{i}$ is a function of some set of properties such as surface gravity and impactor velocity. Thus, there is a set of measured data points, listed as a vector $\mathbf{d}$, that are presumed to be dependent on some unknown set of model parameters, $\mathbf{m}$. In a general sense, this functional dependence can be expressed as $\mathbf{f}(\mathbf{d}, \mathbf{m})=\mathbf{0}$. All of the techniques discussed in this paper require that this functional dependence must be expressed in a way that separates model from data, so that

$$
\mathbf{d}=\mathbf{g}(\mathbf{m})
$$

where $\mathbf{g}$ is a vector function. It is easiest to work with linear vector functions, so that $\mathbf{d}$ is just $\mathbf{m}$ multiplied by some matrix $\mathbf{G}$, or

$$
\mathbf{d}=\mathbf{G m}
$$

As we demonstrate in the specific examples later in the paper, in some cases nonlinear $\mathbf{g}$ can be made linear through a change in variable, working in log-log space, or some other mathematical technique. If the model and data can be put into the linear form, then generally the goal from there is to estimate $\mathbf{m}$ from $\mathbf{d}$; in other words, invert Eq. (2) so that $m$ is isolated.

We can begin the model evaluation by looking at the matrix $\mathbf{G}$. If $\mathbf{G}$ is such that every different $\mathbf{m}$ produces a unique $\mathbf{d}$ and every possible $\mathbf{d}$ can be produced by some choice of $\mathbf{m}$, then the model we 
have chosen is exactly determined. For example, exactly one line can always be fit through any two points. In this case, $\mathbf{G}$ would be square and invertible mathematically. For the exactly determined model, it is a foregone conclusion that the data can be exactly produced by a particular choice of model parameters, and the only means of evaluating the model is whether those model parameters and the model itself make sense. Consider as an example a model where the onset diameter of central peak craters is a planet's gravity multiplied by an arbitrary constant that is different for each planet. Obviously, an arbitrary constant can be chosen for each planet so that the data are always exactly fit; but unless there is some physical basis for the arbitrary constant, its value and the model are essentially meaningless. An exactly determined model can be thought of as transferring the data values into a different set of units.

If $\mathbf{G}$ is such that many different $\mathbf{m}$ can produce the same $\mathbf{d}$, then the inversion for $\mathbf{m}$ is said to be underdetermined. For example, an infinite number of lines can be fit through a single point. For the underdetermined case, evaluation of the model must be essentially independent of the data. For example, if projectile mass, velocity, and impact angle are inputs into a model that predicts the volume of melt for a particular crater, then creating values for mass, velocity, and impact angle for an incoming meteoroid that result in a match to the estimated melt volume says nothing about the validity of the model being used. In simple problems, the underdetermined case occurs when $\mathbf{G}$ has more columns than rows, or $\mathbf{m}$ has more elements than $\mathbf{d}$. In summary, the validity of the model cannot be assessed with inversion techniques for the underdetermined and exactly determined cases.

However, if $\mathbf{G}$ is such that only a subset of possible d's can be produced by all choices of $\mathbf{m}$, then the model is considered overdetermined. For example, of all possible sets of three points, only certain subsets can be fit by the equation of a line. In the overdetermined case, the inversion provides some means of testing the model. A model can be ruled out as invalid if a set of model parameters cannot be found that satisfactorily reproduces the observed set of data. The nature of the inversion is in part based on what criteria define a satisfactory reproduction of the data. In this paper, we seek solutions that minimize the square of the difference between the model results and the data. This is known as a least-squares inversion. In a simple least-squares inversion, the model parameters that best reproduce the data are of the form (Menke, 1989)

$$
\mathbf{m}_{e s t}=\left[\mathbf{G}^{\mathrm{T}} \mathbf{G}^{-1} \mathbf{G}^{\mathrm{T}} \mathbf{d}\right.
$$

Equation (3) gives equal value to each data point; however, it may be decided that it is more important to fit certain data points than others. In this case, the data can be weighted in the inversion so that

$$
\mathbf{m}_{e s t}=\left[\mathbf{G}^{\mathrm{T}} \mathbf{W G}\right]^{-1} \mathbf{G}^{\mathrm{T}} \mathbf{W d}
$$

where $\mathbf{W}$ is a square diagonal matrix of weighting values for each data point. In this paper, we will weight the data by the inverse of its standard deviation, so that data points known with the smallest amount of error are weighted most heavily.

Equation (4) and many other linear inversion formulae have the form $\mathbf{m}_{\text {est }}=\mathbf{M d}$, where $\mathbf{M}$ is the inversion matrix. If the data are independent of each other so that their covariance is a diagonal matrix $\mathbf{V}$ where each diagonal element is $\sigma_{i}^{2}$, the variance of a data point, then an estimate of the model parameter variance is

$$
\mathbf{V}_{\mathbf{m}}=\mathbf{M V} \mathbf{M}^{\mathrm{T}}
$$

An a priori estimate of the model parameters $<m>$ can be given weight in the inversion so that the output model parameters are reasonably close to this initial estimate. Combining weighting of the $a$ priori estimate with the weighted least-squares inversion gives

$$
\mathbf{m}_{e s t}=<\mathbf{m}>+\left[\mathbf{G}^{\mathrm{T}} \mathbf{W} \mathbf{G}+\epsilon^{2} \mathbf{W}_{\mathbf{m}}\right]^{-1} \mathbf{G}^{\mathrm{T}} \mathbf{W}[\mathbf{d}-\mathbf{G}<\mathbf{m}>] \text { Eq. (6) }
$$

where $\mathbf{W}_{\mathbf{m}}$ is a square diagonal matrix individually weighting each model parameter and $\epsilon^{2}$ provides relative weighting between the data and the a priori estimate. Equation (6) can be used to address the nonlinear case where the problem can be defined in terms of Eq. (1) but not Eq. (2). If the functions in Eq. (1) vary smoothly, then each function can be expanded as a Taylor series where we ignore second and higher order terms, so that

$$
d_{i} \approx g_{i}\left(m_{j}^{0}\right)+\frac{\partial g_{i}}{\partial m_{j}^{0}}\left(m_{j}-m_{j}^{0}\right)
$$

where $m_{j}^{0}$ is some initial estimate of a model parameter that the Taylor series is expanded about. In matrix form, this becomes

$$
\mathbf{d} \approx \mathbf{G}<\mathbf{m}>+\mathbf{G}^{\prime}(\mathbf{m}-<\mathbf{m}>)
$$

where $\mathbf{G}^{\prime}$ is the matrix of partial derivatives. Setting $\Delta \mathbf{d}=\mathbf{d}-\mathbf{G}<\mathbf{m}>$ and $\Delta \mathbf{m}=\mathbf{m}-<\mathbf{m}>$, and utilizing Eq. (6), it is possible to create an iterative method for solving for the best possible estimate of $\mathbf{m}$. In this method, known as the Marquardt-Levenberg method (Levenberg, 1944; Marquardt, 1963), a previous estimate of $\mathbf{m}$ and its model output are used for $<\mathbf{m}>$ and $\mathbf{G}<\mathbf{m}>$ and the matrix of derivatives is used in the inversion, so that the next estimate can be calculated as

$$
\Delta \mathbf{m}=\left[\mathbf{G}^{\mathbf{T}} \mathbf{W} \mathbf{G}^{\prime}+\beta\right]^{-1} \mathbf{G}^{\prime \mathbf{T}} \mathbf{W} \Delta \mathbf{d}
$$

The damping factor $\beta$ generally reduces the step size to stabilize the inversion. Typically iterations are continued until the step size falls below a specified value.

Once the model parameters are estimated, one way to evaluate the model is to compare the prediction error, the difference between model results and the data, with the standard deviation $\sigma$ of the data. Comparison of different models designed to reproduce the same data must take into account the fact that the model with more parameters has an inherent advantage in reproducing the data. This comparison can be performed with the $F$ test (Menke, 1989). The $F$ value is the ratio of $\chi_{v}^{2}$ for each model, where

$$
\chi_{v}^{2}=1 / v \sum_{i=1}^{N} e_{i}^{2} / \sigma_{d i}^{\text {rrue }} ; v=N-M
$$

$e$ is prediction error, $N$ is the number of data points, $M$ is the number of model parameters, and $v$ is the number of degrees of freedom. Note that $\chi_{v}^{2}$ becomes smaller with lower prediction errors but larger with more model parameters. The ratio,

$$
F=\chi_{v_{1}}^{2} / \chi_{v_{2}}^{2}
$$

can be compared with standard statistical tables to determine whether a significant difference exists between the two models.

\section{HYDROSTATIC PRESSURE VERSUS STRENGTH}

A particularly illustrative example of the inversion technique is to test the role of hydrostatic pressure in the complex crater formation process. Many conceptual models of complex crater formation envision collapse of a parabolic transient cavity when the hydrostatic pressure at the cavity's base exceeds some measure of rock strength (e.g., Hartmann, 1972; Melosh, 1977, 1982; Grieve et al., 1981). If proportional growth is assumed so that the depth/diameter 
ratio of a transient cavity is invariant in all cases, then the crater diameter for collapse will depend on the factor $c / \rho g$, where $\rho$ is surface density, $g$ is surface gravitational acceleration, and $c$ is some measure of crustal strength. For the terrestrial planets, the crustal strength and density are thought to be similar, leading to inverse gravity scaling or a dependence on the factor $1 / g$. In particular, Pike (1980a, 1988) was able to show that a wide variety of collapse features (e.g., inflection of depth-diameter data, central peak onset, peak ring onset) on the Moon, Mercury, Mars, and Earth can be fit with a line of $1 / \mathrm{g}$ slope. Since that work, the equivalent morphological data has been collected for Venus and several icy satellites, and this greatly expands the range of available data for all three variables in the factor $c / \rho g$. This additional data also makes it feasible to use the inversion approach to test the importance of the factor $c / \rho g$ in the complex cratering process. A qualitative, forward-modeling approach to this test was taken in Herrick and Phillips (1994), and a preliminary inversion was performed in Herrick et al. (1996).

To test the importance of the factor c/og, we assume that all interplanetary differences in crater morphometry are directly controlled by this factor. If the range of values assumed by this factor is small enough, then any dependence can be approximated by an exponential function

$$
O_{j i}=A_{i}\left(\frac{c_{j}}{\rho_{j} g_{j}}\right)^{b_{i}}
$$

where $O_{j i}$ is an observation of type $i$ for planet $j$, and $A$ and $b$ are constants. In log-log space this becomes

$$
(\log O)_{j i}=(\log A)_{i}+b_{i}(\log c)_{j}-b_{i}(\log \rho g)_{j}
$$

and a series of equations in the form of Eq. (1) exists. If $b$ is held constant, then the inversion is linear and Eq. (4) is used for the inversion; but if $b$ is allowed to vary, then the nonlinear form of Eq. (9) must be used. In this paper, we show results from both cases.

The particular measurements we used are the following: complex crater depth, the inflection point of the depth-diameter curve, diameter of central peak onset, diameter of peak ring onset, and diameter of terracing onset. Complex crater depths are functions for each planet rather than the single value necessary for Eq. (12). Therefore, we used the depth of a $30 \mathrm{~km}$ diameter crater, a diameter at which craters on all the planets considered are central-peak craters. There are at least three distinct measurements that could be considered the onset diameter: the diameter of the smallest crater with a particular feature, the diameter of the largest crater without a feature, and the median diameter, where there are as many craters below the median diameter with the feature as there are craters above the median without the feature. We refer to these measurements as the minimum, maximum, and median onset diameters, respectively. Which type of measurement should be used is dependent on both the particulars of a model and subjective evaluation of the data. For example, one could take the position that the median diameter is most representative of what would occur on an ideal planet and that the spread about this value represents natural scatter. In this case, the median value would be most appropriate, but the scatter should be similar on all the bodies so any of the three measurement types would be okay. From a different point of view, one might consider, for example, the minimum onset of central peaks to be the true onset diameter, and larger craters without central peaks have had their peaks covered by later crater infilling. Obviously other scenarios can be constructed.
Table 1 shows the values we used in our various analyses along with the appropriate references. The surface gravities $\left(\mathrm{m} \mathrm{s}^{-2}\right)$ of the planets for which data were collected for this study are: Venus, 8.87; Mercury, 3.78; Mars, 3.72; Moon, 1.62; Ganymede, 1.43; Titania, 0.372; Rhea, 0.285; Ariel, 0.251; Dione, 0.224; and Mimas, 0.079 . We attempted to maximize the chance of comparing apples with apples by generally using data for fresh craters occurring in a single, hopefully nonlayered terrain type. For example on the Moon, fresh highlands craters were used and on Venus, fresh craters on the volcanic plains were used. Also, crater fields, crater chains, highly oblique impacts, and other oddities were not included in the data used. Because terrestrial morphological data is both scant and must be reconstructed with an implicit model from a heavily eroded surface, we have chosen not to use any terrestrial data in our study. As stated above, an inversion can be weighted by the error in the data. Calculating errors for the depth measurements and the inflection point of the depth-diameter curve is a standard statistical problem, and in many cases the error values are given in the stated references. However, determining error bars for the onset of a feature is not as straightforward. To estimate the error, we first assume that our crater data set is a typical sampling of a hypothetical data set containing an infinite number of craters. Our sample set of measurements contains $N$ craters, where $N$ is the number of craters with a feature and below the median diameter plus those craters without a feature and above the median diameter. An infinite number of sampled data sets of our fictitious infinite crater set should have a normal distribution of median values. If we assume that our observed data is a typical sampling of the hypothetical crater population of infinite size, then the $1 \sigma$ error bounds for the median value can be estimated by taking the difference of the two data values $\sqrt{N}$ samples away from the median. Using a similar logic for minimum and maximum onset diameters, the $1 \sigma$ error is defined by taking the difference of the observed value's diameter and the diameter for sample $i$ away from observed value such that

$$
1-\left(\frac{N-i}{N}\right)^{N}>0.68
$$

For example, consider the observed data for Venus regarding the onset of central peaks. Figure I shows the data between the minimumsized crater with a central peak and the maximum-sized crater without one. One hundred ninety-seven craters fall between the minimumsized crater with a central peak, at $8.6 \mathrm{~km}$ diameter, and the maximumsized crater without a central peak, $22.6 \mathrm{~km}$ in diameter. There are 27 craters above $14.8 \mathrm{~km}$ diameter without a central peak and 27 craters below $14.8 \mathrm{~km}$ with a central peak, making $14.8 \mathrm{~km}$ (1.17 in $\log$ space) the median diameter and $N$ equal to 54 . Counting eight samples each way (rounding up from $\sqrt{54}$ ) gives an error for the median in $\log$ space of $0.5(\log 15.7-\log 13.5)$, or 0.033 . With $N=$ $54, i$ must equal 2 for $\mathrm{Eq}$. (14) to hold. Counting two samples away, the error for the maximum is $(\log 22.6-\log 20.5)=0.042$ and for the minimum is $(\log 9.6-\log 8.6)=0.048$. If a reference in Table 1 did not provide error estimates or the data necessary to estimate errors, for the purposes of the inversion we assumed an error in $\log -\log$ space of 0.1 , or $\sim 25 \%$.

We performed a series of linear inversions with $b$ fixed at one unless otherwise stated. A target density of $3000 \mathrm{~kg} \mathrm{~m}^{-3}$ was assumed for the terrestrial planets and moons and $1000 \mathrm{~kg} \mathrm{~m}^{-3}$ for the icy satellites. For the first inversion, we simply tried to fit all of the data: rim-floor depth of a $30 \mathrm{~km}$ crater, the inflection point in the depth-diameter curve, and all three onset diameter measurements for 
TABLE I. Data and model results for inversions involving hydrostatic pressure dependence.

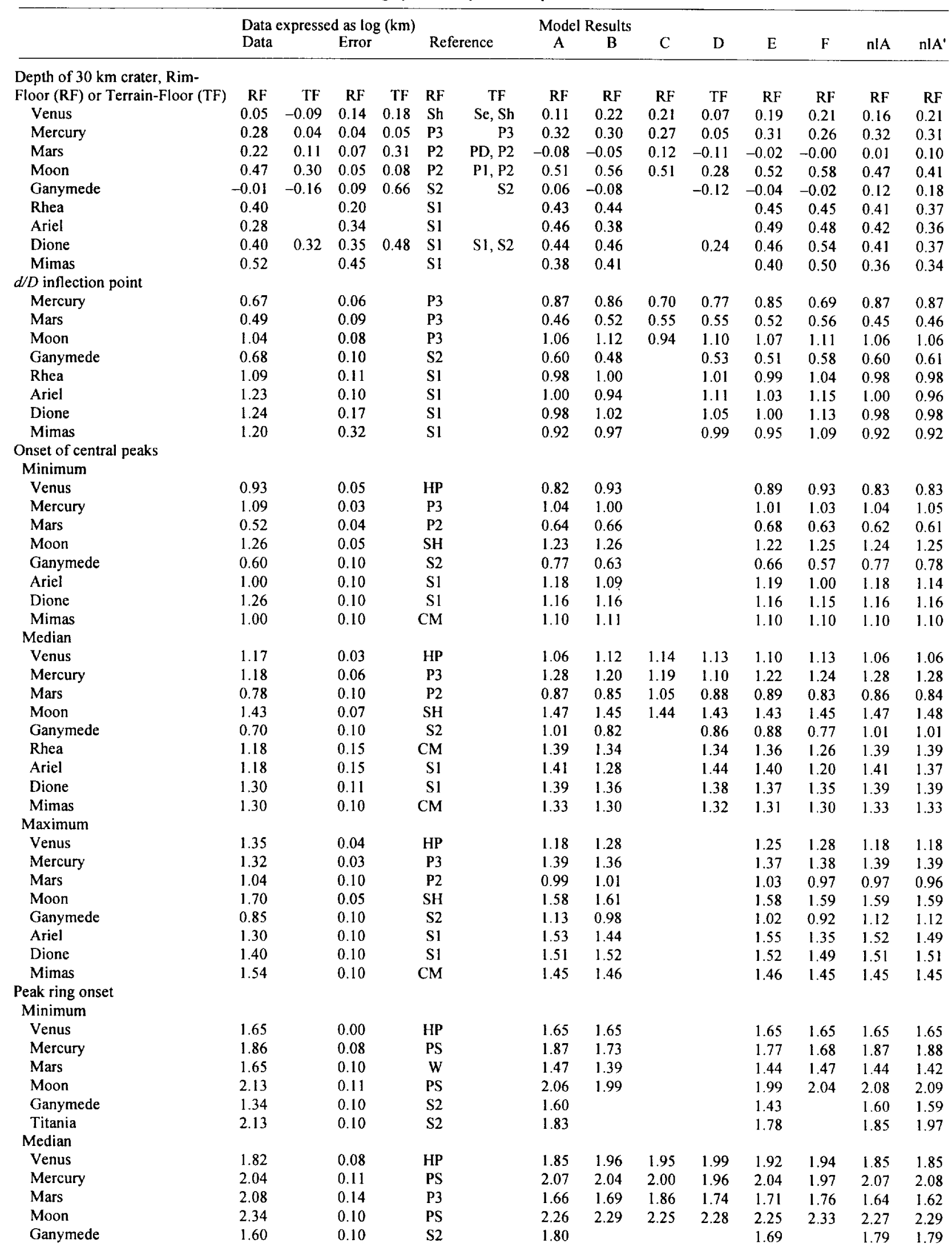


central peaks, peak rings, and terraces. For the icy satellites, we used the onset of central pit craters as data for the onset of peak rings, as some authors (Melosh, 1982; Passey and Shoemaker, 1982; Schultz, 1988) have considered central pits and peak rings to be formed by similar processes. The form of Eq. (13) is such that only relative strengths for the planets can be solved for, and we arbitrarily fixed the lunar value of $c$ to be 10 ( 1 in $\log$ space). To force the central pit onset data to be overconstrained, we assigned Ariel and Titania the same strengths. This inversion has 70 data values and 20 parameters we are inverting for ( $A$ 's and $c$ 's in Eq. (13)). Tables 1 and 2 summarize the results for this inversion, which we will call Model $A$. Some of the fits of model to data are shown graphically in Fig. 2. This inversion was surprisingly successful. With the model parameters that were inverted for, the forward model is able to fit 51 of the 70 data

TABLE 1. Continued.

\begin{tabular}{|c|c|c|c|c|c|c|c|c|c|c|c|c|c|c|}
\hline & \multicolumn{4}{|c|}{ Data expressed as $\log (\mathrm{km})$} & \multirow{2}{*}{\multicolumn{2}{|c|}{ Reference }} & \multicolumn{2}{|c|}{ Model Results } & \multirow[b]{2}{*}{$\mathrm{C}$} & \multirow[b]{2}{*}{$\mathrm{D}$} & \multirow[b]{2}{*}{$\mathrm{E}$} & \multirow[b]{2}{*}{$\mathrm{F}$} & \multirow[b]{2}{*}{$\mathrm{n} \mid \mathrm{A}$} & \multirow[b]{2}{*}{$\mathrm{nlA}^{\prime}$} \\
\hline & Data & & Error & & & & A & B & & & & & & \\
\hline \multicolumn{15}{|l|}{ Maximum } \\
\hline Venus & 1.88 & & 0.03 & & HP & & 1.87 & & & & 1.88 & & 1.87 & 1.87 \\
\hline Ganymede & 1.72 & & 0.10 & & $\mathrm{~S} 2$ & & 1.82 & & & & 1.66 & & 1.81 & 1.81 \\
\hline $\begin{array}{l}\text { Terracing onset: } D \text { or } d \text { at onset } \\
\text { Minimum }\end{array}$ & $D$ & $d$ & $D$ & $d$ & $D$ & $d$ & $D$ & & & & $d$ & & $D$ & $D$ \\
\hline Venus & 0.93 & -0.12 & 0.02 & 0.14 & HP & $\mathrm{HP}, \mathrm{Sh}$ & 1.04 & & & & 0.09 & & 1.04 & 1.04 \\
\hline Mercury & 1.26 & 0.22 & 0.00 & 0.04 & SH & SH, P3 & 1.26 & & & & 0.21 & & 1.26 & 1.26 \\
\hline Mars & 0.83 & -0.04 & 0.06 & 0.07 & $\mathrm{P} 2$ & P2 & 0.85 & & & & -0.12 & & 0.83 & 0.81 \\
\hline Moon & 1.20 & 0.38 & 0.19 & 0.05 & SH & SH, P2 & 1.44 & & & & 0.42 & & 1.46 & 1.46 \\
\hline Ganymede & 1.30 & -0.09 & 0.10 & 0.09 & S2 & S2 & 0.99 & & & & -0.14 & & 0.98 & 0.98 \\
\hline \multicolumn{15}{|l|}{ Median } \\
\hline Venus & 1.11 & -0.06 & 0.03 & 0.14 & HP & $\mathrm{HP}, \mathrm{Sh}$ & 1.13 & & & & 0.16 & & 1.13 & 1.13 \\
\hline Mercury & 1.41 & 0.28 & 0.10 & 0.04 & SH & SH, P3 & 1.35 & & & & 0.28 & & 1.34 & 1.34 \\
\hline Mars & 0.90 & -0.02 & 0.10 & 0.07 & $\mathrm{P} 2$ & P2 & 0.94 & & & & -0.05 & & 0.92 & 0.90 \\
\hline Moon & 1.49 & 0.46 & 0.03 & 0.05 & SH & SH, P2 & 1.54 & & & & 0.49 & & 1.55 & 1.55 \\
\hline Ganymede & 1.60 & 0.05 & 0.10 & 0.09 & S2 & S2 & 1.08 & & & & -0.07 & & 1.07 & 1.07 \\
\hline \multicolumn{15}{|l|}{ Maximum } \\
\hline Venus & 1.29 & -0.01 & 0.04 & 0.14 & $\mathrm{HP}$ & $\mathrm{HP}, \mathrm{Sh}$ & 1.36 & & & & 0.24 & & 1.36 & 1.36 \\
\hline Mercury & 1.69 & 0.40 & 0.23 & 0.04 & SH & SH, P3 & 1.57 & & & & 0.36 & & 1.58 & 1.58 \\
\hline Mars & 1.04 & 0.04 & 0.10 & 0.07 & $\mathrm{P} 2$ & P2 & 1.17 & & & & 0.03 & & 1.15 & 1.14 \\
\hline Moon & 1.62 & 0.50 & 0.21 & 0.05 & SH & $\mathrm{SH}, \mathrm{P} 2$ & 1.76 & & & & 0.57 & & 1.78 & 1.79 \\
\hline Ganymede & 1.78 & 0.12 & 0.10 & 0.09 & $\mathrm{~S} 2$ & $\mathrm{~S} 2$ & 1.31 & & & & 0.01 & & 1.30 & 1.30 \\
\hline rms error: data & & & & & & & 0.13 & 0.14 & 0.08 & 0.20 & 0.13 & 0.14 & 0.13 & 0.13 \\
\hline rms error: model & & & & & & & 0.17 & 0.13 & 0.11 & 0.14 & 0.14 & 0.10 & 0.17 & 0.17 \\
\hline
\end{tabular}

References: P1 = Pike, 1977; P2 = Pike, 1980a; P3 = Pike, 1988; S1 = Schenk, 1989; S2 = Schenk, 1991; Sh = Sharpton, 1994; Se = Sharpton et al., $1994 ; \mathrm{PD}=$ Pike and Davis, 1984; HP = Herrick and Phillips, 1994; CM = Chapman and McKinnon, 1986; W = Wood, 1980; PS = Pike and Spudis, 1987; SH = Smith and Hartnell, 1978.

central peak $\$+\$+4+20$

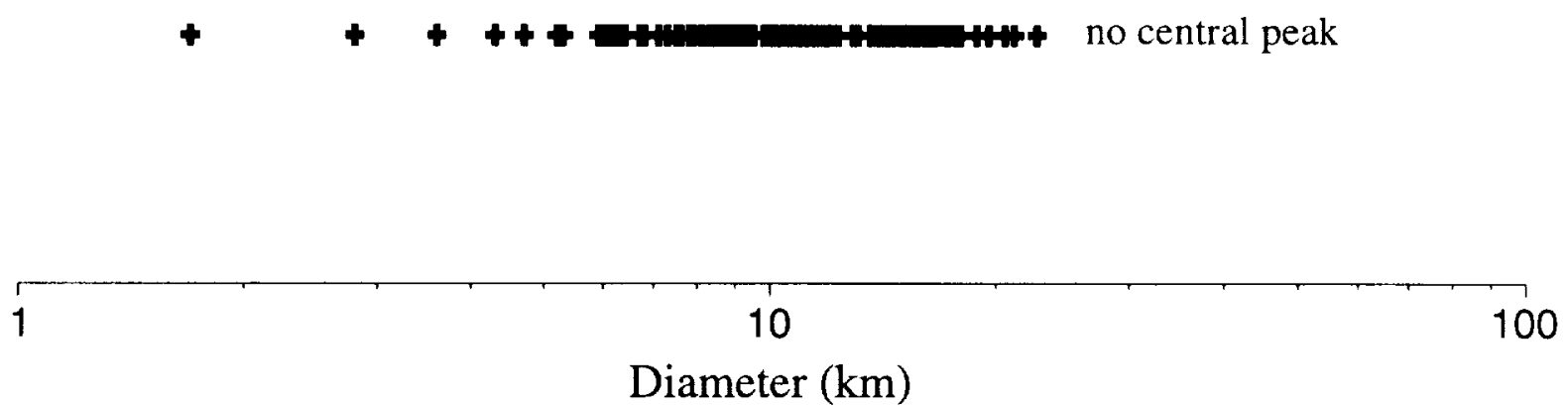

FIG. I. Raw data for the onset of central peaks for craters on Venus. Graph shows the diameters of craters with and without a central peak. The minimum onset diameter is the diameter of the smallest crater with a central peak, or $8.6 \mathrm{~km}$. The maximum onset diameter is the diameter of the largest crater without a central peak, or $22.6 \mathrm{~km}$. The median onset diameter is where the number of craters below the median and with a central peak equals the number above the median and without a central peak, or $14.8 \mathrm{~km}$. 
TABLE 2. Model parameters and their formal errors for inversions involving hydrostatic pressure.

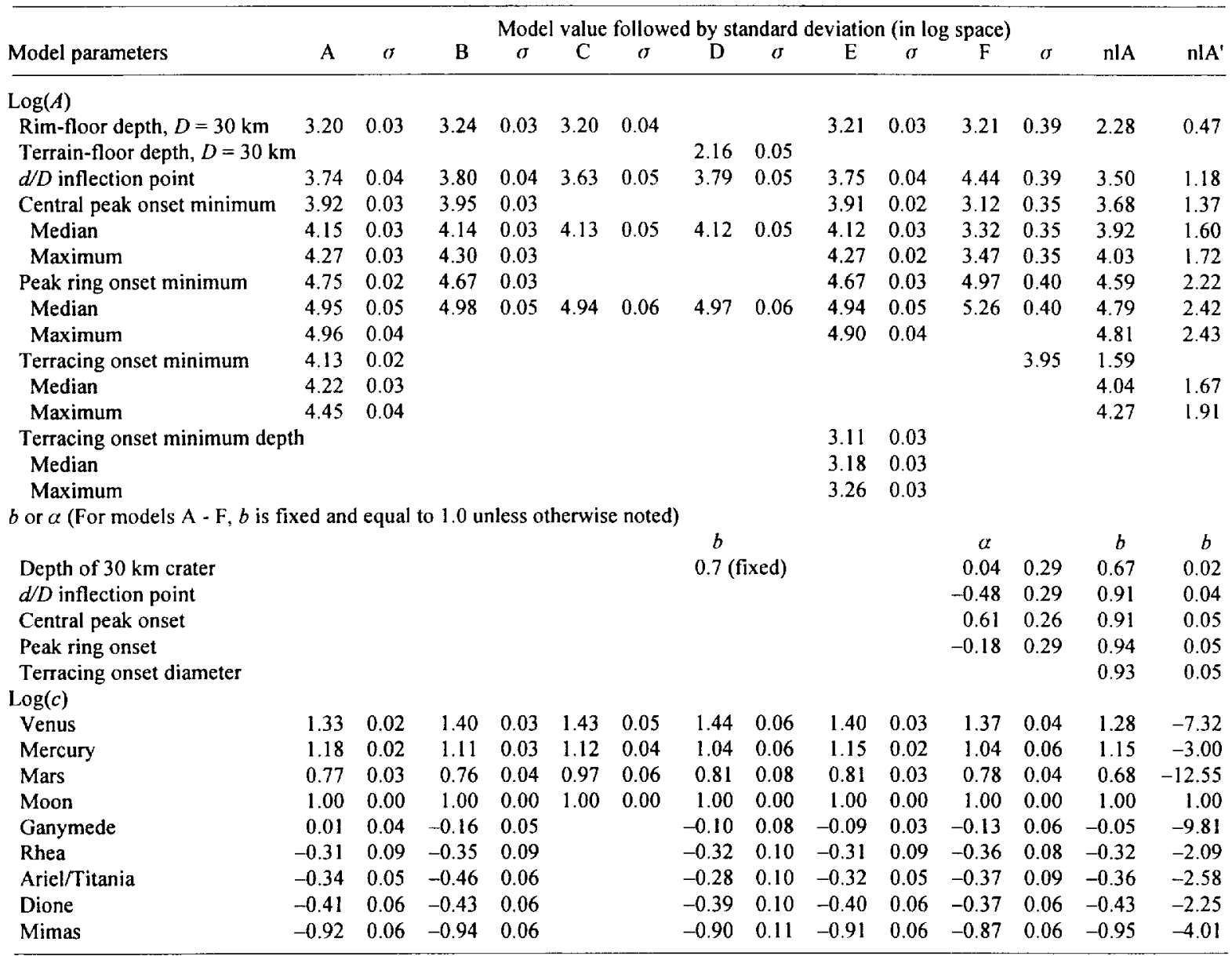

points within $2 \sigma$ of the observed value, and 61 of 70 within $3 \sigma$. The rms error (in log space) for the model output is $0.17 \mathrm{vs} .0 .13$ for the actual data, so overall the inversion did not produce an acceptable fit to the data. The worst fits of model to data occur for the onset of terracing and the onset of central pits (treated as peak rings) on the icy satellites. The majority of the terracing data is not fit within $1 \sigma$, and four of the fifteen data values are not fit within $3 \sigma$ of the data. Of the four data values for the onset of central pits on Ganymede and Titania, only one was fit within acceptable error bars. These poor fits suggest that the model we used, Eq. (13) with $b$ fixed at 1, was inappropriate for these two data sets. It is encouraging that the model outputs for $c$, the strength parameter, seem reasonable. The icy satellites as a group have strengths an order of magnitude less than those for the terrestrial planets. Mars, which may have water in the crust, has a relative strength half that of the Moon. Mercury, which superficially appears to most resemble the lunar highlands, has a value of $c$ that is $50 \%$ larger than the Moon's. Venus has a dry crust that may not be as highly fractured as the lunar or Mercurian crust, and its calculated relative strength is roughly double the Moon's. For the icy satellites, Ariel, Rhea, Dione and Titania have strengths roughly half that of Ganymede's, and the strength of Mimas is about one-tenth that of Ganymede's.

There are many logical variations on this first model that can be tested, and we summarize a few here. We ran another inversion identical to Model A, except it did not include the terracing data or the central pit onset data. The results for this inversion, Model $\mathrm{B}$ in Tables 1 and 2, were similar to those for Model A and indicate that including the terracing and central pit data did not adversely affect the rest of the inversion in Model A. The Model $B$ results have a slightly lower rms error than the data because the terracing and central pit data were excluded, and all but three of the data points were fit within $3 \sigma$. For the test labeled Model $\mathrm{C}$ in Tables 1 and 2, we used only data for the rocky planets and the following measurements: rim-floor depth of a $30 \mathrm{~km}$ crater, the inflection point in the depthdiameter curve, and the median onset diameters for central peaks and peak rings. The input for Model $\mathrm{C}$ was a small subset of that for Model $\mathrm{A}$, but the output results were remarkably similar, and the same general trend in planetary strengths was found. Without the inclusion of icy satellites, the rms error for the data was substantially lower than the error for the data used in Model A. Thus, even though Model $\mathrm{C}$ had a lower rms model output error than Models A or B, the model output does not have an rms error lower than the observed data. All of the data were fit within $3 \sigma$ with Model $\mathrm{C}$.

Recently McKinnon et al. (1997) stated that a $g^{-0.7}$ trend was more logical for the depth trend for a single diameter, and they also preferred to use terrain-floor depth data rather than the rim-floor depths that are commonly used. We ran a set of four tests where we ran through the possible combinations of using terrain-floor depth $v s$. rim-floor depth and forcing a $(c / \rho g)^{1} v s$. $(c / \rho g)^{0.7}$ trend for the depths. The measurements used for the inversions were depth of a $30 \mathrm{~km}$ cra- 

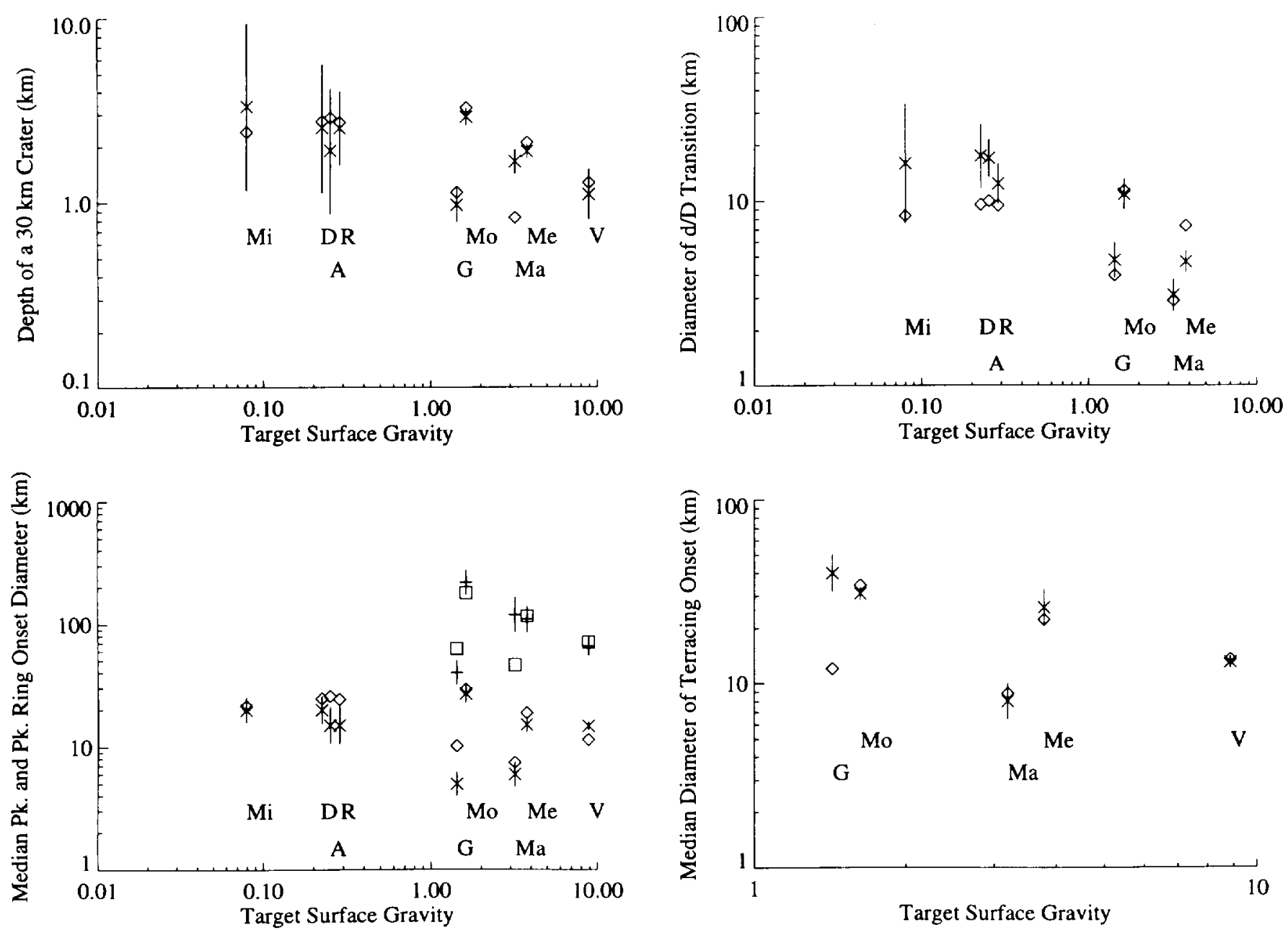

FIG. 2. Graphical representation of the data and model results for Model A in Tables 1 and 2. Data is shown as x's (horizontal lines for median peak ring onset) with vertical lines representing $1 \sigma$ error bars. Model results are shown as diamonds (squares for median peak ring onset). Data and values are plotted vs. target surface gravity. Data and results for Mars have been plotted at a slightly lower than actual surface gravity to allow the points to be distinguished from the Mercurian data and results.

ter, the inflection point in the depth-diameter curve, and the median onset diameters for central peaks and peak rings. These four inversions all yielded similar results that were also consistent with Models A and B. Figure 3 compares, for all four inversions, the calculated model trends $v s$. the depth data. The results for one of these, the one using terrain-floor depths and forcing a $(c / \rho g)^{0.7}$ depth trend, are shown as Model D in Tables 1 and 2. Negligible differences were found between model fits using terrain-floor $v s$, rim-floor depths, but the relative strengths of Venus, Mercury, and Mars were slightly lower if terrain-floor depths were used. Almost identical model fits were found between models using a $(c / \rho g)^{0.7} v s$. a $(c / \rho g)^{1}$ trend, and the primary model parameter that changed between these two models was the constant $A$ in Eq. (12) in response to the forced change in $b$.

We also performed several nonlinear inversions similar to models A-D but allowing $b$ to be a variable for each measurement type. For the nonlinear inversions, an initial estimate of model parameters must be given as a starting point for the inversion, and damping of individual steps may be important. The nonlinear inversion can be thought of as starting at a specified point on a surface of model solution errors and using local slopes to end up in a local minimum that minimizes error. Ideally there is only one local minimum that is the absolute minimum, and that minimum is reached from any starting point. Unfortunately, as we discuss below, that is not the case for the inversions performed here. A judicious choice of the damping factor will get the inversion to a local minimum in a few steps without overshooting that minimum. We found that the most effective technique was to start with minimal damping and automatically increase damping as necessary to ensure that each step produced a model output with a lower error than the previous step. We ceased iterating when the average step became less than $5 \times 10^{-4}$ the average model parameter.

Model nlA in Tables 1 and 2 shows the results for a nonlinear inversion that used the results of linear Model $A$ as a starting set of parameters. We allowed $b$ to vary, but required $b$ to be the same for the minimum, maximum, and median of each measurement type. Thus, Models A and nlA are fitting the same data, but Model nlA is nonlinear and has five additional parameters (the $b$ 's). This particular inversion converged to a minimum error solution with fairly minor changes in the model parameters from the starting values. In particular, the calculated $b$ values stayed near 1.0 except for the $30 \mathrm{~km}$ crater depths, which inverted for a slope of 0.69 . This would seem to validate the linear dependence on $c / \rho g$ except for the $(c / \rho g)^{0.7} \mathrm{de}$ pendence on depth suggested by McKinnon et al. (1997). However, 

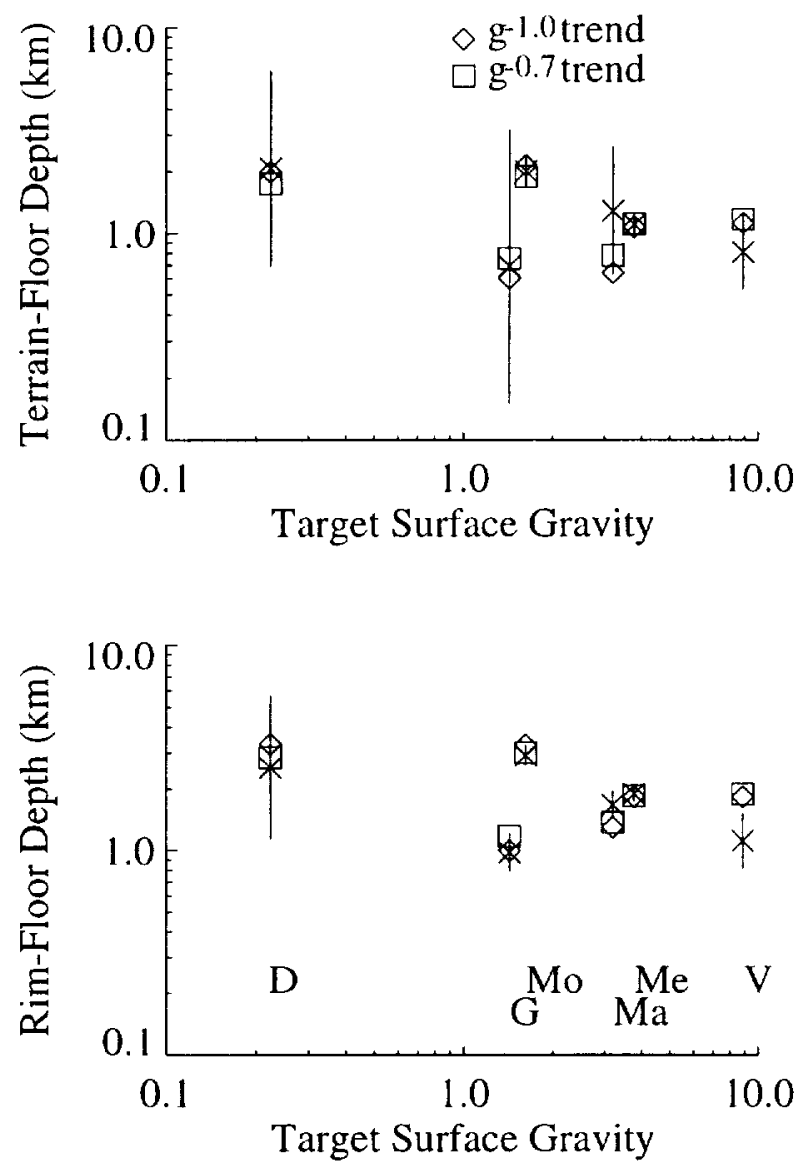

FIG. 3. Crater depth $v s$. target gravity for $30 \mathrm{~km}$ central peak craters on six different planetary bodies. Results are from identical inversions (Model D in Tables 1 and 2) using the four possible combinations of rim-floor depths or terrain-floor depths and forcing a $g^{-0.7}$ or $g^{-1.0}$ trend. The top plot shows results vs. data for inversions using terrain-floor depths with the two gravity trends and the bottom plot shows similar results for inversions using rim-floor depths. Data is shown as X's with vertical lines representing l $\sigma$ error bars, and model results are shown as diamonds $\left(g^{-1.0}\right.$ trend $)$ and squares $\left(g^{-0.7}\right.$ trend)

there was less than a percent difference in data error between the outputs of the linear and nonlinear models. In other words, allowing $b$ to vary essentially gained nothing in terms of the overall model being able to fit the data.

We also found that the solution set of model parameters is nonunique. Model $n l A^{\prime}$ in Tables 1 and 2 shows the results from an inversion with a different set of starting parameters. In this case, the solution evolved to a set of functions with nearly flat slopes $(b \sim 0)$ and still achieved the same fit of model results to observed data. Two characteristics of the observed data account for the nonuniqueness of inversion results. First, when plotted on a log-log graph of measurement value $v s$. surface gravity, most of the observed data sets are roughly parallel. Proportionality constants (the $A$ 's) can be solved for that have the effect of collapsing several data sets into one line. Second, we expect the strengths of the planetary bodies to have roughly the same trend as the gravity. The rocky planets have higher surface strengths and higher surface gravities than the icy satellites. Therefore, higher or lower slopes (the $b^{\prime} s$ ) can be counteracted by less or more dramatic strength variations. Additional nonlinear inversions were performed corresponding to the data sets used for some of the other linear inversions, and results were similar to those obtained in Models nIA and nlA'.

$F$ tests showed that in no case was the model error reduced by using the nonlinear inversions more than could be attributed to simply adding parameters to the model. In other words, a simple linear dependence on the factor $c / \rho g$ works pretty well at matching most of the data we used except for the onset of terracing data. While the nonlinear inversion results were not an improvement over the linear model, they also showed that a linear dependence is not the only acceptable model for fitting the data.

Model A shows that terracing onset data do not reasonably fit a c/ $\rho g$ dependence. Some previous works (e.g., Pike, 1980b; Herrick and Phillips, 1994) have suggested that the poor fit for terracing onset might be because terracing is a late-stage process that occurs after final crater depth has largely been determined. To test this idea in a primitive way, we assume that the complex crater depth $d$, rather than diameter, for the onset of terracing is dependent on the factor $c / \rho g$. We performed another inversion with Model A modified by substituting crater depth for crater diameter in the data for onset of terracing. These results are shown as Model $\mathrm{E}$ in Tables 1 and 2. The reduction in error is dramatic. For Model E, 58 of 70 model parameters are fit within $2 \sigma$ and 66 of 70 are fit within $3 \sigma$. Overall the rms model error is $<10 \%$ greater than the rms data error. Thus, our inversion results support the hypothesis that terracing occurs when final crater depth exceeds a constant multiple of $c / \rho g$.

\section{ACOUSTIC FLUIDIZATION}

A more specific model of complex crater formation that has been proposed is the acoustic fluidization model of Melosh (1982). The basic premise of the acoustic fluidization model is that a hemispherical region containing the transient cavity becomes fluidized by acoustic noise. The fluidized material behaves as a Bingham plastic, a material that behaves as a solid below a yield stress $c$ but flows as a fluid when differential stresses exceed this strength. Melosh (1982) assumes a fixed viscosity $\eta$ for the fluidized material, which then behaves as a damped, oscillating wave that freezes when stresses fall below $c$. The shape of the wave when it freezes determines whether the crater has a central peak, a peak ring, or multiple rings. Initial collapse occurs if

$$
\rho g H / c \geq 5
$$

where $H$ is the depth of the transient cavity, and collapse becomes deep-seated if the inequality is $>8$ to 15 . Melosh (1982) assumes a depth/diameter ratio of 1:5 for the transient cavity, so collapse occurs when

$$
\rho g D / c \geq 25
$$

The break in the depth-diameter function is considered by Melosh (1982) to be the best estimate of when collapse occurs, so the diameters of these breaks and Eq. (16) as an equality can be used to invert for $c$. When collapse is deep-seated, the final depth $d$ of the crater should be a constant independent of diameter but determined by $\mathrm{c} / \rho g$ so that

$$
d=K c / \rho g
$$

where $K$ is a constant that should be between 8 and 15 . We can use the depth of a $30 \mathrm{~km}$ diameter crater and Eq. (17) to invert for $K$ and $c$. Through a series of approximations, Melosh (1982) determines that the number of zero crossings that occur in the oscillating wave is, 
TABLE 3. Results from acoustic fluidization inversions

\begin{tabular}{|c|c|c|c|c|c|c|c|c|c|}
\hline & \multicolumn{3}{|c|}{$\begin{array}{c}\text { Approach } 1 \\
\text { data as } \log (\mathrm{m})\end{array}$} & \multicolumn{3}{|c|}{$\begin{array}{c}\text { Approach } 2 \\
\text { data as } \ln (\mathrm{m})\end{array}$} & \multirow[b]{2}{*}{ Parameters } & \multirow[t]{2}{*}{$\underset{(\log )}{\text { App. } 1}$} & \multirow[t]{2}{*}{$\underset{(\ln )}{\text { App. } 2}$} \\
\hline & Data & error & Results & Data & error & Results & & & \\
\hline \multicolumn{7}{|l|}{$d / D$ inflection point } & \multicolumn{3}{|l|}{ Yield stress $(\mathrm{Pa})$} \\
\hline Mercury & 3.67 & 0.06 & 3.73 & 8.45 & 0.06 & 33.60 & Venus & 6.53 & 26.20 \\
\hline Mars & 3.49 & 0.09 & 3.62 & 8.04 & 0.09 & 34.10 & Mercury & 6.37 & 25.20 \\
\hline Moon & 4.04 & 0.08 & 3.97 & 9.30 & 0.08 & 35.00 & Mars & 6.26 & 26.00 \\
\hline Ganymede & 3.68 & 0.10 & 3.57 & 8.48 & 0.10 & 23.60 & moon & 6.24 & 25.70 \\
\hline Rhea & 4.09 & 0.11 & 4.05 & 9.43 & 0.11 & 14.90 & Ganymede & 5.33 & 15.10 \\
\hline Ariel & 4.23 & 0.10 & 4.19 & 9.74 & 0.10 & 11.70 & Rhea & 5.10 & 5.45 \\
\hline Dione & 4.24 & 0.17 & 4.17 & 9.77 & 0.17 & 14.60 & Ariel & 5.19 & 1.96 \\
\hline Mimas & 4.20 & 0.32 & 4.13 & 9.68 & 0.32 & 21.20 & Dione & 5.12 & 4.87 \\
\hline Depth of a $30 \mathrm{~km}$ crater & & & & & & & Mimas & 4.63 & 11.50 \\
\hline Venus & 3.05 & 0.14 & 3.05 & 7.01 & 0.14 & 10.20 & Viscosity (Pa s) & & \\
\hline Mercury & 3.28 & 0.04 & 3.25 & 7.54 & 0.04 & 8.60 & Venus & 9.34 & $1.1 \times 10^{4}$ \\
\hline Mars & 3.22 & 0.07 & 3.15 & 7.42 & 0.07 & 9.58 & Mercury & 9.17 & $9.5 \times 10^{3}$ \\
\hline Moon & 3.47 & 0.05 & 3.49 & 7.98 & 0.05 & 8.75 & Mars & 8.37 & $3.2 \times 10^{3}$ \\
\hline Ganymede & 2.99 & 0.09 & 3.09 & 6.88 & 0.09 & -0.78 & moon & 9.56 & $1.4 \times 10^{4}$ \\
\hline Rhea & 3.40 & 0.20 & 3.57 & 7.84 & 0.20 & -11.40 & Ganymede & 7.86 & $8.3 \times 10^{2}$ \\
\hline Ariel & 3.28 & 0.34 & 3.72 & 7.55 & 0.34 & -14.60 & Rhea & 7.14 & $8.6 \times 10^{1}$ \\
\hline Dione & 3.40 & 0.35 & 3.70 & 7.84 & 0.35 & -12.00 & Aricl & 7.63 & $8.0 \times 10^{1}$ \\
\hline Mimas & 3.52 & 0.45 & 3.66 & 8.11 & 0.45 & -5.61 & Dione & 7.28 & $1.5 \times 10^{2}$ \\
\hline \multicolumn{4}{|c|}{ Median central peak onset } & \multicolumn{3}{|c|}{$N \times N$ for cent. peak onset } & Mimas & 6.67 & $9.3 \times 10^{1}$ \\
\hline Venus & 4.17 & 0.03 & 4.28 & 1.00 & 0.15 & $7.7 \times 10^{-2}$ & K & 0.92 & -9.02 \\
\hline Mercury & 4.18 & 0.06 & 4.44 & 1.00 & 0.30 & $4.1 \times 10^{-3}$ & & & \\
\hline Mars & 3.78 & 0.10 & 4.45 & 1.00 & 0.59 & -0.48 & & & \\
\hline Moon & 4.43 & 0.07 & 4.81 & 1.00 & 0.35 & 0.17 & & & \\
\hline Ganymede & 3.70 & 0.10 & 4.42 & 1.00 & 0.62 & -2.68 & & & \\
\hline Rhea & 4.18 & 0.15 & 4.18 & 1.00 & 0.90 & 1.11 & & & \\
\hline Ariel & 4.18 & 0.15 & 4.18 & 1.00 & 0.90 & -0.16 & & & \\
\hline Dione & 4.30 & 0.11 & 4.30 & 1.00 & 0.59 & 2,09 & & & \\
\hline Mimas & 4.30 & 0.10 & 4.30 & 1.00 & 0.52 & -1.96 & & & \\
\hline \multicolumn{4}{|l|}{ Median peak ring onset } & \multicolumn{6}{|c|}{$\mathrm{N} \times \mathrm{N}$ for peak ring onset } \\
\hline Venus & 4.82 & 0.08 & 4.23 & 4.00 & 0.71 & 7.50 & & & \\
\hline Mercury & 5.04 & 0.11 & 4.23 & 4.00 & 1.06 & 77.40 & & & \\
\hline Mars & 5.08 & 0.14 & 3.68 & 4.00 & 1.52 & 51.90 & & & \\
\hline Moon & 5.34 & 0.10 & 4.61 & 4.00 & 0.97 & 36.00 & & & \\
\hline Ganymede & 4.60 & 0.10 & 3.88 & 4.00 & 1.00 & 50.00 & & & \\
\hline rms error: data & & & 0.17 & & & 0.56 & & & \\
\hline rms error: model & & & 0.43 & & & 21.83 & & & \\
\hline
\end{tabular}

See Table 1 for appropriate references for data values.

$$
N=\frac{1}{\pi}\left[\frac{3}{4}\left(\frac{g \rho^{2} L^{3}}{\eta^{2}}\right)-1\right]^{1 / 2} \ln \left(\frac{1}{2 \sqrt{3}} \frac{\rho g H}{c} \frac{a}{L}\right)
$$

where $L$ is the radius of the fluidized region and $a$ is the radius of the transient cavity. We use Melosh's (1982) assumptions that $L$ is the also the radius of the final crater and that it is equal to $1.5 \mathrm{a}$. Equation (18) becomes

$$
\begin{aligned}
& N=\frac{1}{\pi}\left[\frac{k_{1} D^{3}}{\eta^{2}}-1\right]^{1 / 2} \ln \left(\frac{k_{2} D}{c}\right) \\
& k_{1}=\frac{3 g \rho^{2}}{32} \\
& k_{2}=\frac{2 \rho g}{45 \sqrt{3}}
\end{aligned}
$$

For a given planet, $N=1$ at the onset diameter of central peaks and $N=2$ at the onset diameter of peak rings.
We would like to use our measurements and Eqs. (16), (17), and (19) to invert for each planet's viscosity (in Pa s) and yield stress (in Pa). However, Eq. (19) is not in the form required by Eq. (1) with data and parameters separated. It is impossible to isolate the data values of the onset diameters $D$ in Eq. (19) from the model parameters. We tried two approaches to getting around this problem. In the first approach, we held one of the two terms in Eq. (19) fixed during each inversion step and then recalculated that term between steps. We chose to hold the second term fixed so that for each step we could define a third constant

$$
k_{3}=\left[\frac{N \pi}{\ln \left(k_{2} D / c\right)}\right]^{2}+1
$$

so that now Eq. (19) can be rewritten as

$$
D^{3}=k_{3} \eta^{2} / k_{1}
$$

or in log-log space

$$
\log D=\frac{1}{3}\left(\log k_{3}+2 \log \eta-\log k_{1}\right)
$$


In summary, for the first approach we used log-log versions of Eqs. (16) and (17) along with Eq. (22) to invert depth-diameter transitions, depth data, central peak onsets, and peak ring onsets for the yield stress and viscosity parameters. The inversion has 31 observations to invert for 19 parameters.

In the inversion, $k_{3}$ must be calculated before each step, and this value depends on the value of $D$ used. We ran two inversions, one where the observed diameters were used and the other where $D$ is calculated from Eq. (22) using parameters and model results from the previous iteration. There were no significant differences in the results from either approach, and Table 3 summarizes the results where the observed diameters were used to calculate $k_{3}$. The inversion was unsatisfactory in that no set of model parameters was able to successfully match the data within the data error bars. When taken back out of log space, the data has an rms error of $\sim 50 \%$ while the model produces errors of $\sim 170 \%$, or the model results are typically a factor of 2.7 greater or less than the observed data. The principal failure of the model is that no viscosity values could be found so that the model could match both central peak onsets and peak ring onsets. Examination of Eq. (18) shows that to first order, the model predicts that the ratio of onset diameters for peak rings $v$ s. central peaks should be $2^{2 / 3}$, or $\sim 1.6$, while observed ratios range from about 4 to 20 . Consequently, the best the model can do is choose viscosity values that produce onset diameters intermediate between the observed values for central peaks and peak rings.

In the second approach, we used Eq. (18) directly with $N$ instead of $D$ as the data value we were solving for. We squared Eq. (18) to stabilize the inversion. The data values $D$ were treated as constants in the inversion, but the percent error in $D$ was used as the percent error in $N^{2}$. Because Eq. (18) involves a natural log term, we used $\ln$-In versions of Eqs. (16) and (17) to invert the depth-diameter transitions and depth data. As the results in Table 3 show, this second approach was even less successful than the first approach. In the first approach, the yield stresses $c$ were calculated independently of $\eta$ and were not affected by the problem with the ratio between onset diameters for central peaks and peak rings. In the second approach, the only way the inversion could produce a large difference in $N$ with small differences in $D$ was to choose large strength values that made the natural log term in Eq. (19) at or below zero where the natural $\log$ function is rapidly varying.

Melosh (1982) obtained a fit of model to data by allowing the viscosity to be different for central peak onset and peak ring onset. In this case, the inversion is exactly determined so that the viability of the model must be evaluated by some other means than the model's ability to fit the data. A discussion of the physical viability and the geologic evidence for acoustic fluidization in a crater is beyond the scope of this paper. It is interesting to note that the basic mathematics in the acoustic fluidization model should be appropriate for any conceptual model of complex crater formation that involves Bingham fluid collapse of a hemispherical shell of material. Any such model must have the property of either decreasing $L$ relative to $a$ with increasing crater diameter or increasing $\eta$ with increasing diameter. This would seem to rule out collapse with the hemispherical cavity defined by exposure to a specified shock pressure, as that would pre$\operatorname{dict} L$ increasing relative to $a$ (e.g., Melosh, 1989).

\section{NONPROPORTIONAL GROWTH AND IMPACT VELOCITY EFFECTS}

Some authors have advocated that the onset and formation of certain properties of complex craters are a result of nonproportional growth or a change in the shape of the excavation cavity with increasing crater diameter. In particular, Schultz (1988) has used extrapolation of small-scale impact experiments to argue for shallowing of transient crater diameter above a crater diameter dependent on $v^{1.17} / \mathrm{g}$, where $v$ is impactor velocity. He estimates that both the depth-diameter transition and the onset of peak rings should be proportional to the same parameters, so that

$$
D_{l 1,2}=k_{1,2} \frac{v^{1.17}}{g}\left(\frac{\rho_{t}}{\rho_{p}}\right)^{0.22}\left(\frac{s_{l}^{2}}{s_{p}}\right)^{0.83}\left[1+\left(\frac{\rho_{p}}{\rho_{f}}\right)^{0.5}\right]^{-0.83}
$$

where $s$ is sound speed, the subscripts $t$ and $p$ denote target and projectile, and the subscripts 1,2 indicate the equations for depth-diameter transition and peak ring onset, respectively. Schultz (1988) also includes an equation for peak ring diameter relative to crater diameter, but the predicted and observed interplanetary variations are less than the error bars on the data, so we did not use that data in our inversion. For a given planet, we expect that the highest impact velocities will generally be associated with cometary impactors that we expect to have relatively low densities and sound velocities. We can carry this logic into the inversion by associating the maximum possible impact velocity with the diameter of the largest crater without a peak ring, the minimum possible impact velocity with the diameter of the smallest crater with a peak ring, and the median impact velocity with the median onset diameter of peak rings and the depthdiameter transition.

For the inversion, we estimated all parameters in Eq. (23) except for $k_{1,2}$ and inverted for these two constants in log-log space. For the Moon, Mercury, and Mars, we assumed a target density of $2900 \mathrm{~kg}$ $\mathrm{m}^{-3}$ and sound speed of $3 \mathrm{~km} / \mathrm{s}$; for Venus, we assumed a target density of $2900 \mathrm{~kg} \mathrm{~m}^{-3}$ and sound speed of $4.5 \mathrm{~km} / \mathrm{s}$; and for the icy satellites, we assigned a target density of $1000 \mathrm{~kg} \mathrm{~m}^{-3}$ and sound speed of $1.6 \mathrm{~km} / \mathrm{s}$. For the terrestrial planets, we assumed that the minimum and median velocity impactors were stony asteroids and assigned them a density of $2500 \mathrm{~kg} \mathrm{~m}^{-3}$ and sound speed of $3 \mathrm{~km} / \mathrm{s}$, and the maximum velocity impactors were assumed to be cometary and were assigned a density of $1000 \mathrm{~kg} \mathrm{~m}^{-3}$ and sound speed of 1 $\mathrm{km} / \mathrm{s}$. For the icy satellites, all impactors were assumed to be cometary.

Table 4 shows the impactor velocities used in the inversion, the constants $k_{1,2}$ resulting from the inversion, and the model results compared to the data. The minimum impactor velocity is simply the escape velocity of the target body. The maximum impactor velocity is also calculated theoretically using the orbital and escape velocities of the sun and the pertinent planets and moons. Median impactor velocities for the inner planets are from Schultz (1988); and for Ganymede, the Shoemaker and Wolfe (1982) value for short-period comets is used. For the remainder of the icy satellites, we use as a median velocity the simple approximation of $\sqrt{3}$ times the orbital velocity of the planet orbited by the satellite. This approximation assumes an impacting population of long-period comets and ignores the minor acceleration provided by the gravity wells of the planet and satellite. All of the velocity values used are speculative, but the general trend they define should be accurate. Other than approximately defining the general trend by virtue of the inverse gravity term in Eq. (23), the model results simply do not match the data. The rms model error of 0.65 corresponds to the model results typically being a factor of 4.5 greater or less than the data. 
TABLE 4. Results from nonproportional growth inversions.

Density and velocity parameters used in inversion

Impactor velocities and corresponding impactor density and sound speed Target density

\begin{tabular}{|c|c|c|c|c|c|c|c|c|c|c|c|}
\hline & & & & & & & & & & and sou & d speed \\
\hline & $\underset{\mathrm{km} / \mathrm{s}}{\operatorname{Min} v}$ & $\underset{\mathrm{kg} \mathrm{m}^{-3}}{\rho_{p}}$ & $\begin{array}{c}s_{p} \\
\mathrm{~km} / \mathrm{s}\end{array}$ & $\begin{array}{c}\text { Med. } v \\
\mathrm{~km} / \mathrm{s}\end{array}$ & $\begin{array}{c}\rho_{p} \\
\mathrm{~kg} \mathrm{~m}^{-3}\end{array}$ & $\underset{\mathrm{km} / \mathrm{s}}{s_{p}}$ & $\underset{\mathrm{km} / \mathrm{s}}{\operatorname{Max} .} v$ & $\begin{array}{c}\rho_{p} \\
\mathrm{~kg} \mathrm{~m}^{-3}\end{array}$ & $\underset{\mathrm{km} / \mathrm{s}}{\mathrm{s}_{p}}$ & $\begin{array}{c}\rho_{t} \\
\mathrm{~kg} \mathrm{~m}^{-3}\end{array}$ & $\begin{array}{c}s_{t} \\
\mathrm{~km} / \mathrm{s}\end{array}$ \\
\hline Venus & 10.4 & 2500 & 3 & 27 & 2500 & 3 & 86 & 1000 & 1 & 2900 & 4.5 \\
\hline Mercury & 4.3 & 2500 & 3 & 34 & 2500 & 3 & & & & 2900 & 3.0 \\
\hline Mars & 5.0 & 2500 & 3 & 16 & 2500 & 3 & & & & 2900 & 3.0 \\
\hline moon & 2.5 & 2500 & 3 & 22 & 2500 & 3 & & & & 2900 & 3.0 \\
\hline Ganymede & 2.7 & 1000 & 1 & 16 & 1000 & 1 & 48 & 1000 & 1 & 1000 & 1.6 \\
\hline Rhea & & & & 17 & 1000 & 1 & & & & 1000 & 1.6 \\
\hline Ariel & & & & 12 & 1000 & 1 & & & & 1000 & 1.6 \\
\hline Dione & & & & 17 & 1000 & 1 & & & & 1000 & 1.6 \\
\hline Mimas & & & & 17 & 1000 & 1 & & & & 1000 & 1.6 \\
\hline Titania & 0.8 & 1000 & 1 & & & & & & & 1000 & 1.6 \\
\hline
\end{tabular}

Data and inversion results expressed as $\log (\mathrm{km})$

\begin{tabular}{|c|c|c|c|c|c|c|c|}
\hline rsion results e & Data & Error & Results & & Data & Error & Results \\
\hline$d / D$ inflection point & \multicolumn{7}{|c|}{ Peak ring: median onset } \\
\hline Mercury & 0.67 & 0.06 & 0.70 & Venus & 1.82 & 0.08 & 2.13 \\
\hline Mars & 0.49 & 0.09 & 0.32 & Mercury & 2.04 & 0.11 & 1.87 \\
\hline Moon & 1.04 & 0.08 & 0.85 & Mars & 2.08 & 0.14 & 1.50 \\
\hline Ganymede & 0.68 & 0.10 & 0.66 & Moon & 2.34 & 0.10 & 2.02 \\
\hline Rhea & 1.09 & 0.11 & 1.39 & Ganymede & 1.60 & 0.10 & 2.28 \\
\hline Ariel & 1.23 & 0.10 & 1.26 & \multicolumn{4}{|l|}{ Peak ring: maximum onset } \\
\hline Dione & 1.24 & 0.17 & 1.49 & Venus & 1.88 & 0.03 & 3.27 \\
\hline Mimas & 1.20 & 0.32 & 1.94 & Ganymede & 1.72 & 0.10 & 2.84 \\
\hline Peak ring: minimum onset & & & & \multicolumn{3}{|l|}{ rms data error } & 0.12 \\
\hline Venus & 1.65 & 0.00 & 1.65 & \multicolumn{3}{|l|}{ rms model error } & 0.12 \\
\hline Mercury & 1.86 & 0.08 & 0.82 & \multirow{5}{*}{$\begin{array}{l}\text { Parameters } \\
\log \left(k_{1}\right) \\
\log \left(k_{2}\right)\end{array}$} & & & \multirow{5}{*}{$\begin{array}{l}-3.69 \\
-2.06\end{array}$} \\
\hline Mars & 1.65 & 0.10 & 0.91 & & & & \\
\hline Moon & 2.13 & 0.11 & 0.91 & & & & \\
\hline Ganymede & 1.34 & 0.10 & 1.39 & & & & \\
\hline Titania & 2.13 & 0.10 & 1.34 & & & & \\
\hline
\end{tabular}

We also tried a model that tested a more general dependence on impact velocity. We modified the linear form of Eq. (12) to allow for a possible exponential dependence on velocity, or

$$
O_{j i}=A_{i} \frac{c_{j}}{\rho_{j} g_{j}} v_{j}^{\alpha_{i}}
$$

where $\alpha$ is an unknown exponential. In $\log -\log$ space, this expression becomes

$$
(\log O)_{j i}=(\log A)_{i}+(\log c)_{j}-(\log \rho g)_{j}+\alpha_{i}(\log v)_{j}
$$

which is still linear if we assume we know the impact velocities. We ran an inversion with the same data set as Model B but with additional parameters defined by the last term in Eq. (25). We used only median impact velocities and had a separate $\alpha$ for depth of a $30 \mathrm{~km}$ crater, the d/D inflection point, onset of central peaks, and onset of peak rings. The same a was used for the minimum, median, and maximum onset diameters, so a total of four parameters were added to the inversion of Model B.

Our initial inversion was unstable. We decided to perform another inversion with some minimal weighting given to an a priori estimate to stabilize the solution. For a priori estimates, we used the model parameter results of Model B and varied the $\alpha$ values from 0 to 2 . We found that providing a uniform weighting of the a priori esti- mate of $\sim 0.1 \%$ was all that was necessary to stabilize the solution, and the model results using an a priori estimate had an rms error within $3 \%$ of the unconstrained inversion. Model $\mathrm{F}$ in Tables 1 and 2 shows the results with an a priori estimate of 1.0 for the $\alpha$ values. In our solutions, only the $\alpha$ value for onset of central peaks consistently had an absolute value significantly different from zero, and an $F$ test comparison between Models $F$ and $B$ indicates that the reduction in error from Model $B$ to Model $F$ is not statistically significant. We conclude that there is no evidence for an impact velocity dependence for the measurements used in Models B and F, at least not in the form of Eq. (25). We emphasize that our results do not imply that varying impact velocity has no effect on the volume of the excavation cavity but merely support the notion that the shape of the excavation cavity is velocity-independent. The observables we used in our inversions are for craters formed by hypervelocity impacts in the gravity-dominated regime, and, thus, our results do not apply to differences between subsonic and hypervelocity impacts or to craters formed in the strength-dominated regime.

\section{DISCUSSION}

We have inverted crater morphometry data to test general and specific models for complex crater formation. A general dependence on hydrostatic pressure $v s$. strength, or the factor $c / \rho g$, works remarkably well for most of the data. The trend in planetary crustal strengths that is inferred from the inversions is consistent with our expectations: Venus has the highest crustal strength, Mercury and the Moon have 

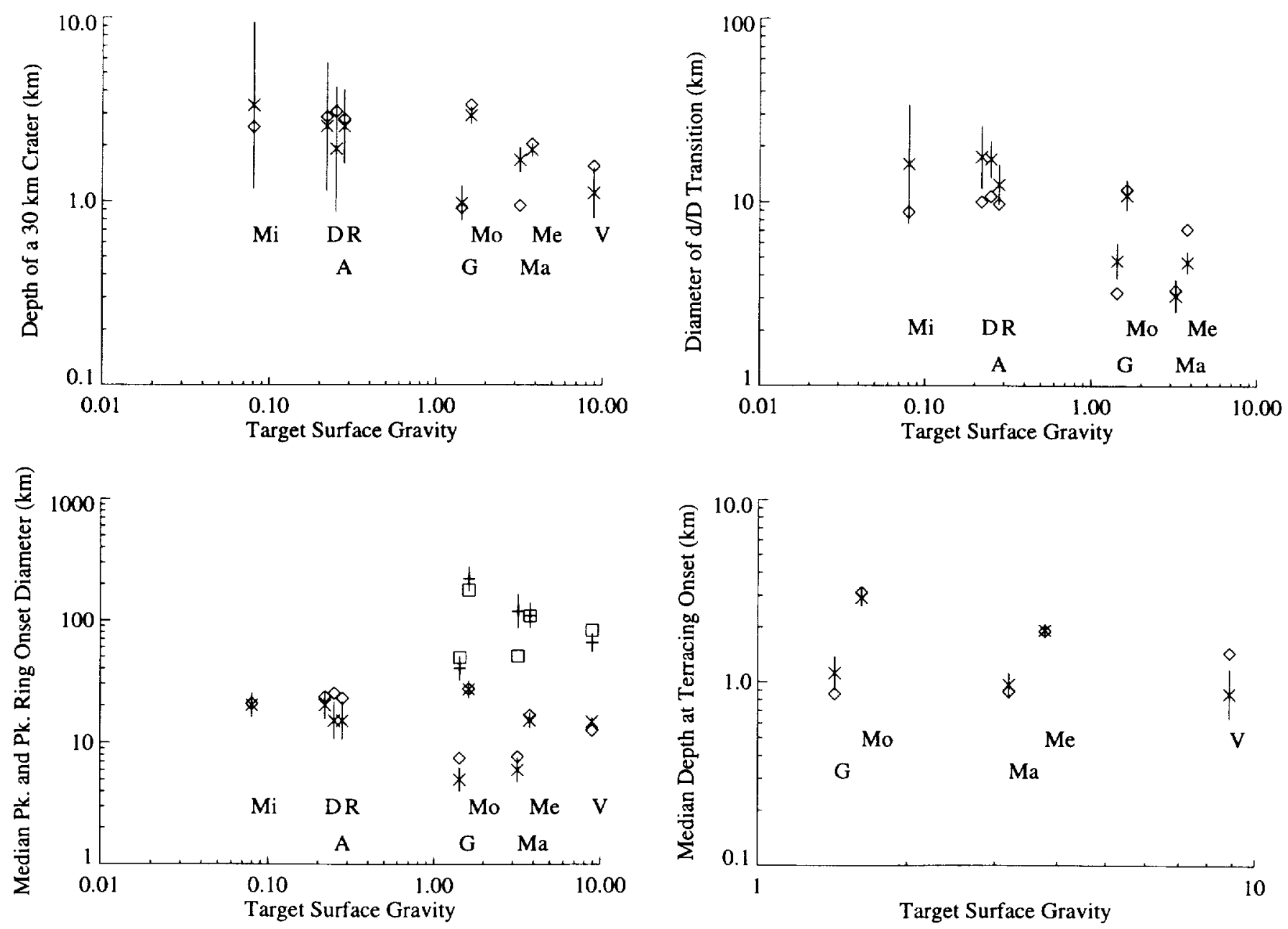

FIG. 4. Similar to Fig. 2 but using model results from Model $\mathrm{E}$ in Tables 1 and 2.

slightly lower strengths because of a more fractured crust, Mars has a still lower strength perhaps because of water in the crust, and the icy satellites are an order of magnitude weaker than the terrestrial planets. The acoustic fluidization model of Melosh (1982) did not fit the data unless the number of model variables was increased until the model was even determined; for even determined models, the fit of model to data is guaranteed and is not an indicator of the model's validity. The nonproportional growth formulation of Schultz (1988) did not fit the data, and no dependence on impact velocity in general was found.

A dependence on the factor $c / \rho g$ does not reproduce all of the data. The diameter onset of terracing does not follow a $c / p g$ trend but the depth onset of terracing does (Model A vs. Model E). This may indicate that terracing is a late-stage process that occurs after the final depth of the crater has been determined, an idea first suggested by Pike (1980b) using only data from Mars, the Moon, and Mercury. While the central pit onset data did not seem to fit the peak ring onset data trend in Model A, in Model $\mathbf{E}$ the slight change in relative crustal strengths results in three of four model results for central pit onset falling within error bars for the data. Figure 4 shows graphically the model results $v s$. data for model $\mathrm{E}$. Perhaps what stands out most in Fig. 4 is that Mars consistently does not fall on the trend produced by the other planets. This may indicate that there is something unique about the modification stage of complex crater formation on Mars, perhaps as a result of significant portions of both rock and water ice in the crust.
It is difficult to determine exactly what is unique about Mars because we don't really understand why a dependence on c/pg fits the data so well. While a dependence on hydrostatic pressure may seem to make sense for the initial collapse, the final crater depth, and the depth onset of terracing, there is no obvious reason why the trend applies to peak ring onset. Furthermore, why collapse occurs at all is somewhat puzzling, as interior slopes in simple craters are generally below the angle of repose (Melosh, 1977). Our results from the nonlinear models further complicate the issue by indicating that the dependence on $c / \rho g$ need not be linear; exaggerating or minimizing strength differences can offset a lower or higher exponential dependence on $c / \rho g$. However, because the trends are all roughly parallel in $\log -\log$ space, values of $b$ are required to be roughly similar for all the measurement types, perhaps within \pm 0.5 .

It is perhaps indicative of how little is really understood about complex crater formation that very few models in the literature make quantitative testable predictions of interplanetary variations in crater shape, and those few do not fit the data. Our inversions confirm the long held qualitative belief that hydrostatic pressure vs. crustal strength is important in complex crater formation and in fact seem to indicate that $c / \rho g$ is the dominant factor. A quantitative physical model that explains in detail this apparent importance has yet to be developed. The general inversion techniques we present can be used as powerful tools for testing future models. 
Acknowledgments-This work was supported by NASA grant NASW-4574 to the Lunar and Planetary Institute. We thank Eileen Ryan and Bill I lartmann for useful comments. This is LPI Contribution 931

Editorial handling: W. K. Hartmann

\section{REFERENCES}

ALEXOPOULOS J S AND MCKINNON W B (1994) Large impact craters and basins on Venus, with implications for ring mechanics on the terrestrial planets. In Large Meteorite Impacts and Planetary Evolution (eds. B. O. Dressler, R. A. F. Grieve, and V. L. Sharpton) pp. 29-50. GSA Special Paper 293, Boulder, Colorado.

BALDWIN R. B. (1949) The Face of the Moon. University of Chicago Press, Chicago, Illinois. $239 \mathrm{pp}$

ChapMAN C. R. AND MCKINNON W. B. (1986) Cratering of planetary satellites. In Satellites (eds. J. A. Burns and M. S. Matthews), pp. 492-580. Univ. Arizona Press, Tucson, Arizona.

Grieve R. A. F., Robertson P. B. AND DENCE M. R. (1981) Constraints on the formation of ring impact structures, based on terrestrial data. In Multiring Basins Proc Lunar Planet Sci. Conf 12A, 37-57.

HALE W. AND HEAD J. W. (1979) Central peaks in lunar craters: Morphology and morphometry. Proc. Lunar Planet. Sci. Conf. 10th, 2623-2633.

HALE W. AND HEAD J. W. (1980) Central peaks in Mercurian craters: Comparisons to the moon. Proc Lunar Planet. Sci. Conf. 11th, 2191-2205.

HALE W. AND HEAD J. W. (1981) Central peaks in Martian craters: Comparison to the moon and Mercury (abstract). Lumar Planet. Sci. 12, 386-388.

HARTMANN W. K. (1972) Interplanet variations in scale of crater morphologyEarth, Mars, Moon. Icarus 17, 707-713

HERRICK R. R. AND PHILLIPS R. J. (1994) Implications of a global survey of Venusian impact craters. Icarus 111, 387-416.

Herrick R. R., Sharpton V. I., Mal. IN M. C., Lyons S. N. ANd FeEly K. (1996) Morphology and morphometry of impact craters. In Venus I/ (eds. S. W. Bougher, D. M. Hunten and R. J. Phillips) pp. 1015-1046. Univ. Arizona Press, Tucson, Arizona

LEVENBERG K. (1944) A method for the solution of certain nonlinear problems in least squares. Quat. Appl. Math. 2, 164-168.

LINES L. R. AND TREITEL S. (1984) Tutorial: A review of least-squares inversion and its application to geophysical problems. Geophys. Prospecting 32, 159-186.

MALIN M. C. AND DzURISIN D. (1978). Modification of fresh crater landforms: Evidence from the moon and Mercury. J. Geophys. Res. 83 233-243.

MAROUARDT D. W. (1963) An algorithm for least squares estimation of non-linear parameters. J. Soc. Indust. Appl. Math. 11,431-441.

MCKINNON W. B., ZAIINLE K. J., IVANOV B. A. AND MELOSH H. J. (1997) Cratering on Venus: Models and observations. In Venus II (eds. S. W. Bougher, D. M. Hunten and R. J. Phillips). Univ. Arizona Press, Tucson, Arizona, 969-1014.

MELOSH H. J. (1977) Crater modification by gravity: $A$ mechanical analysis of slumping. In Impact and Explosion Cratering (eds. D. J. Roddy,
R. O Pepin and R. B. Merrill), pp.1245-1260. Pergamon. New York. New York.

Mel.osh H. J. (1982) A schematic model of crater modification by gravity J. Geophys. Res. 87, 371-380.

Melosi H. J. (1989) Impact Cratering Oxford Univ. Press. New York, New York. 245 pp

MENKE W. M. (1989) Geophysical Data Analysis: Discrete Inverse Theory, Revised Edition. Academic Press, San Diego, California. $289 \mathrm{pp}$

PASSEY Q. R. AND SHOEMAKER F. M. (1982) Craters and basins on Ganymede and Callisto: Morphological indicators of crustal evolution. In Satellites of Jupiter (ed. D. Morrison), pp. 379-434. Univ. Arizona Press, Tucson, Arizona.

PIKE R. J. (1977) Size dependence in shape of fresh impact craters on the moon. In Impact and Explosion Cratering (eds. D. J. Roddy, R. O Pepin and R. B. Merrill), pp. 489-509. Pergamon, New York. New York.

PIKE R. J. (1980a) Control of crater morphology by gravity and target type: Mars, Earth, Moon. Proc. Lunar Planet. Sci. Conf. 11th, 2159-21 89.

PIKE R. J. (1980b) Formation of complex impact craters: Evidence from Mars and other planets. Icarus 43, 1-19.

PIKE R. J. (1988) Geomorphology of impact crater on Mercury. In Mercury (eds. F. Vilas, C. R. Chapman and M. S. Matthews), pp. 165-273. Uniy Arizona Press, Tucson, Arizona.

PIKE R. J. AND DAVIS P. A. (1984) Towards a topographic model of Martian craters from photoclinometry (abstract). Lunar Planet. Sci. 18. 645-646.

PIKE: R. J. AND SPUDIS P. D. (1987) Basin-ring spacing on the moon, Mercury, and Mars. Earth, Moon, and Planets 39, 129-194.

SCHENK P. M. (1989) Crater formation and modification on the icy satellites of Uranus and Saturn: Depth/diameter and central peak occurrence. $J$ Geophys. Res. 94, 3813-3832.

SCHENK P. M. (1991) Ganymede and Callisto: Complex crater formation and planetary crusts. J. Geophys. Res. 96, $15635-15664$.

SCHULTZ P. H. (1988) Cratering on Mercury: A relook. In Mercury (eds. F Vilas, C. R. Chapman and M. S Matthews), pp. 274-335. Univ. Arizona Press, Tucson, Arizona.

SHARPTON V. L. (1994) Evidence from Magellan for unexpectedly deep complex craters on Venus. In Large Meteorite Impacts and Planetary Evolution (eds. B. O. Dressler, R. A. F. Grieve and V. L. Sharpton) pp. 19-27. GSA Special Paper 293, Boulder, Colorado

SharPton V. L. , HERriCK R. R. AND KLAUS K. (1994) Topography of Venusian craters and implications for cratering mechanics (abstract). Eos Trans. AGU, Fall Meeting Supplement 75, 413.

SHOEMAKER E. M. AND WOLFE R. F. (1982) Cratering time scales for the Galilean satellites. In Satellites of Jupiter (ed. D. Morrison), pp. 277-339. Univ. Arizona Press, Tucson, Arizona.

SMith E. I. AND HaRTNELl J. A. (1978) Crater size-shape profiles for the moon and Mercury: Terrain effects and interplanetary comparison. Moon and Planets 19, 479-511.

Wood C. A (1980) Martian double ring basins: New observations. Proc. Lunar Planet. Sci Conf. 11 th, 2221-2241.

WOOD C. A. AND ANDERSSON L. (1978) New morphometric data for fresh lunar craters. Proc Lunar Planet Sci Conf 9th, 3669-3689. 


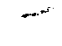

